\title{
Influence of chronic hepatitis $C$ infection on the monocyte-to-platelet ratio: data analysis from the National Health and Nutrition Examination Survey (2009-2016)
}

\author{
Aidan M. Nikiforuk ${ }^{1,2}$, Mohammad Ehsanul Karim ${ }^{1,3}$, David M. Patrick ${ }^{1,4}$ and Agatha N. Jassem ${ }^{2,5^{*}}$
}

\begin{abstract}
Background: Hepatitis C virus (HCV) causes life-threatening chronic infections. Implementation of novel, economical or widely available screening tools can help detect unidentified cases and facilitate their linkage to care. We investigated the relationship between chronic HCV infection and a potential complete blood count biomarker (the monocyte-to-platelet ratio) in the United States.

Methods: The analytic dataset was selected from cycle years 2009-2016 of the National Health and Nutrition Examination Survey. Complete case data- with no missingness- was available for $n=5281$ observations, onehundred and twenty-two $(n=122)$ of which were exposed to chronic HCV. The primary analysis used surveyweighted logistic regression to model the effect of chronic HCV on the monocyte-to-platelet ratio adjusting for demographic and biological confounders in a causal inference framework. Missing data and propensity score methods were respectively performed as a secondary and sensitivity analysis.

Results: In the analytic dataset, outcome data was available for $n=5281$ ( $n=64,245,530$ in the weighted sample) observations of which $n=122$ ( $n=1,067,882$ in the weighted sample) tested nucleic acid positive for HCV. Those exposed to chronic HCV infection in the United States have 3.10 times the odds of a high monocyte-to-platelet ratio than those not exposed (OR=3.10, [95\% Cl: 1.55-6.18]).

Conclusion: A relationship exists between chronic HCV infection and the monocyte-to-platelet ratio in the general population of the United States. Reversing the direction of this association to predict chronic HCV infection from complete blood counts, could provide an economically feasible and universal screening tool, which would help link patients with care.
\end{abstract}

Keywords: Viral hepatitis, Causal inference, Machine learning, Diagnostic screening, Hepacivirus C

\footnotetext{
* Correspondence: agatha.jassem@bccdc.ca

${ }^{2}$ British Columbia Centre for Disease Control Public Health Laboratory,

Virology, Provincial Health Services Authority, Vancouver, British Columbia

V5Z 4R4, Canada

${ }^{5}$ Department of Pathology and Laboratory Medicine, University of British

Columbia, Vancouver, British Columbia V6T 1Z4, Canada

Full list of author information is available at the end of the article
}

(c) The Author(s). 2021 Open Access This article is licensed under a Creative Commons Attribution 4.0 International License, which permits use, sharing, adaptation, distribution and reproduction in any medium or format, as long as you give appropriate credit to the original author(s) and the source, provide a link to the Creative Commons licence, and indicate if changes were made. The images or other third party material in this article are included in the article's Creative Commons licence, unless indicated otherwise in a credit line to the material. If material is not included in the article's Creative Commons licence and your intended use is not permitted by statutory regulation or exceeds the permitted use, you will need to obtain permission directly from the copyright holder. To view a copy of this licence, visit http://creativecommons.org/licenses/by/4.0/ The Creative Commons Public Domain Dedication waiver (http://creativecommons.org/publicdomain/zero/1.0/) applies to the data made available in this article, unless otherwise stated in a credit line to the data. 


\section{What is new}

- Modern techniques in causal inference were applied to understand the relationship between chronic hepatitis $C$ virus infection and the monocyte-toplatelet ratio in complex survey data from the United States of America.

- Chronic hepatitis $C$ virus infection alters a patient's monocyte-to-platelet ratio, previous studies found an association with platelets (thrombocytopenia) but not monocytes at the population level.

- The monocyte-to-platelet ratio could be used as a tool to screen patients for hepatitis $C$ virus diagnostic tests from a complete blood draw.

\section{Introduction}

Hepatitis $C$ virus infects over 71 million people globally causing complex liver disease and more years of life lost than any other pathogen [1]. In 2015, the World Health Organization estimated that approximately half a million people died from $\mathrm{HCV}$-related disease and three times as many were newly infected [2].

Responding to the global HCV epidemic requires country-specific strategies [3]. In the United States, fifteen to $20 \%$ of infections progress to the chronic stage, requiring treatment $[4,5]$. Undiagnosed infection can lead to liver damage, cancer and death [3]. The prognosis of HCV infection has recently improved due to the development of short course, tolerant and effective direct-acting antiviral drugs. Direct-acting antivirals clear chronic $\mathrm{HCV}$ infections at a $90 \%$ rate, making the elimination of the virus plausible for the first time $[3,6]$. Utilizing the full potential of direct-acting antiviral drugs for treatment-based elimination, requires the development of new tools to detect unrecognized cases and link them with care [3].

The diagnosis of chronic $\mathrm{HCV}$ typically involves the administration of two tests. A serological test to detect anti-HCV immunoglobulin, indicates exposure or acute infection. A positive serological result prompts the requisition of a nucleic acid amplification test (NAAT). Unlike serological methods, NAAT differentiates past exposure from acute or chronic infection by detecting viral ribonucleic acid [7]. Biomarkers offer an antigenfree alternative for the detection of a pathological process [8]. Clinical researchers have developed the aspartate aminotransferase-to-platelet ratio index (APRI) as a biomarker of late HCV infection with the purpose of predicting hepatic fibrosis [9]. We postulate that significant differences in patient biomarkers may occur early in the time course of $\mathrm{HCV}$ infection, enabling screening of undiagnosed chronic cases.

The described analysis examines the relationship between chronic HCV infection (exposure) and the monocyte-to- platelet ratio (MPR) (outcome) in subjects from the National Health and Nutrition Examination Survey (NHANES) cycles 2009-2016 [10]. Studies of chronic HCV have identified an association between viral load and monocyte, platelet counts $[11,12]$. Immunological processes support the direction and causality of this relationship. The proliferation of HCV stimulates the innate immune system leading to an increase in the population of monocytes [13]. In a cross-sectional comparison of HCV positive- and negative- patients the percentage of monocytes in a complete blood count increased in response to infection $(\Delta=0.9 \%, P \quad<0.001)$ [14]. Thrombocytopenia (a decreased platelet count) occurs in response to liver disease or interaction between the virus and the innate immune response [11]. In a previous NHANES III study, $13 \%$ of HCV seroconverted patients had a depleted platelet count while only $5 \%$ of negative patients had less than $<175 \times 10^{9}$ platelets per litre [12]. Comparison of this result with other reports $[11,14]$ indicates that the expected effect size of HCV infection on complete blood counts depends on values used to categorize a normal from abnormal count. To limit misclassification bias we composed the MPR from numeric platelet and monocyte counts before categorization by the mean value of $\mathrm{HCV}$ negative patients. We hypothesize that complete blood counts from patients with chronic HCV will have a higher MPR than those not infected. Understanding the relationship between chronic $\mathrm{HCV}$ infection and MPR may allow for screening to occur from complete blood counts, making population level screening more economically feasible.

\section{Methods}

\section{Data, design and study population}

This cross-sectional study investigated the relationship between chronic $\mathrm{HCV}$ infection and MPR from complete blood counts using NHANES survey data cycles 2009 to 2016 [10]. The NHANES survey represents the non-institutionalized civilian population of fifty American states and the district of Columbia through complex sampling design. The design includes primary sampling units, strata and sample weights, a full description of how these measures were derived is available from the Centers for Disease Control and Prevention [15]. Oversampling was conducted for those greater than 80 years of age and identifying as an African American, Asian or Hispanic ethnicity. Survey features were included in the analysis to generalize the findings to the population of the United States [16, 17]. The aggregated medical examination center weights were divided by the duration of the study in two-year survey cycles $(n=4)$ (Additional file 1: Table S1). The University of British Columbia's Policy 89, item 7.10.3 on studies involving human participants and Article 2.2 of the Tri-Council Policy Statement on Ethical Conduct for Research Involving Humans, provide ethical support of the study 
$[18,19]$. NHANES is a publicly available dataset administered by the National Center for Health Statistics, written informed consent was provided by all participants [20].

\section{Analytic sample and variable selection}

In the analytic dataset, we have included the exposure (chronic HCV infection) and outcome (monocyte-toplatelet ratio) of interest, select covariates or potential confounders in the relationship between chronic HCV infection and MPR. The exposure of interest (LBXHCR) categorically measures chronic HCV infection by NAAT where: "1" signals positive, " 2 "- negative, "3"- no seroconversion (lack of HCV specific antibodies) and "." as missing (Additional file 1: Table S1) [15]. Exposure was releveled to "1" as positive and "0" as negative or no seroconversion. The outcome variable (MPR) was constructed from complete blood count measures of monocyte count ("LBDMONO") and platelet count ("LBXPLTSI") (Additional file 1: Table S1) [15]. The monocyte-to-platelet ratio (MPR) was calculated by dividing thousands of monocytes per microliter by thousands of platelets per microliter. The liquid volume measurement was then converted to milliliters. All variables in the analytic dataset were renamed, coded or leveled to ease interpretation (Additional file 1: Table S1).

Covariates were identified from previous population level studies of HCV patients and plotted in a directed acyclic graph (DAG) (Additional file 1: Figure S1) [21, 22]. The selected covariates portray measurements of general health status, social-economic status, biological sex, age, race, marital status, transfusion status, white blood cell count, needle use, average alcohol intake per day, anemia, cancer or diabetes diagnosis (Additional file 1: Figure S1). Exclusion criteria removed subjects with a positive human immunodeficiency virus test result, those pregnant or less than 18 years of age (Fig. 1). After excluding $n=18,895$ observations for missing information on fifteen variables, $n=5281$ individuals with complete case data were identified, of whom $n=112$ had chronic $\mathrm{HCV}$ infection (Table 1, Figure S1). Confounders were selected by the disjunctive cause criterion and included throughout automated variable selection (Additional file 1: Figures S1, S2) [18]. Covariates which did not meet the requirements of the disjunctive cause criterion were selected for inclusion by automated variable selection; therefore, they were only included if they increased the model fit and precision of the estimates.

\section{Statistical analysis}

\section{Transformation of the monocyte-to-platelet ratio}

The monocyte-to-platelet ratio was dichotomized by linear regression [9]. As described by the theory of mean-of-class classification, linear regression was used to find the mean MPR of $\mathrm{HCV}$ negative participants in the complete case dataset $[23,24]$. The continuous numeric MPR variable was then dichotomized to a factor with two levels. Logistic regression and a receiver operator characteristic curve analysis were used to predict $\mathrm{HCV}$ infection by dichotomized MPR for transparency, and to show that we did not choose a biased value for categorization.

\section{Descriptive statistics}

Characteristics of the analytic sample were determined by unweighted survey-sample number and as a weighted sample percentage, representing the population percentage. The sample was stratified by the outcome of interest (MPR), independence was tested between the outcome and covariates via the Rao-Scott Chi-square test for survey design [25].

\section{Statistical inference}

Primary analysis used survey-weighted logistic regression to model the relationship between chronic $\mathrm{HCV}$ and MPR adjusting for known confounders (i.e. age, type 2 diabetes mellitus and needle use) [11, 26-28]. Covariates were chosen through literature review, confounders were selected by the disjunctive cause criterion and use of a directed acyclic graph (DAG) [29, 30]. Missing data and propensity score analyses were performed secondarily and to assess sensitivity of the results.

The primary analysis utilized a survey-weighted logistic regression model to estimate the effect of chronic HCV infection on MPR [17]. Confounders defined by use of a DAG were included in the model. Covariates selected by literature review which did not meet the definition of a confounder were selected for inclusion in the model by backward elimination on the Akaike information criterion (AIC) [31]. Identified confounders were locked in the backward elimination algorithm, so that only covariates which did not meet the definition of a confounder were evaluated for inclusion by automated variable selection (Additional file 1: Figure S2). Collinearity was assessed by the variance inflation factor, with a cutoff of 5 [32]. The primary analysis was performed in the complete case dataset, using the R packages: tidyverse, dataexplorer, jtools, car, tableone, survey, publish, MASS, pROC and caret.

The secondary analysis used multiple imputation by chained equations to provide missing data on the covariate needle use. Under the assumption of missing at random, predictors of needle use were selected by a minimum correlation of $5 \%$ [33]. Missing data were imputed in ten datasets with predictive mean matching, the number of iterations was set to match the percentage missing (12.87). A survey-weighted logistic regression 


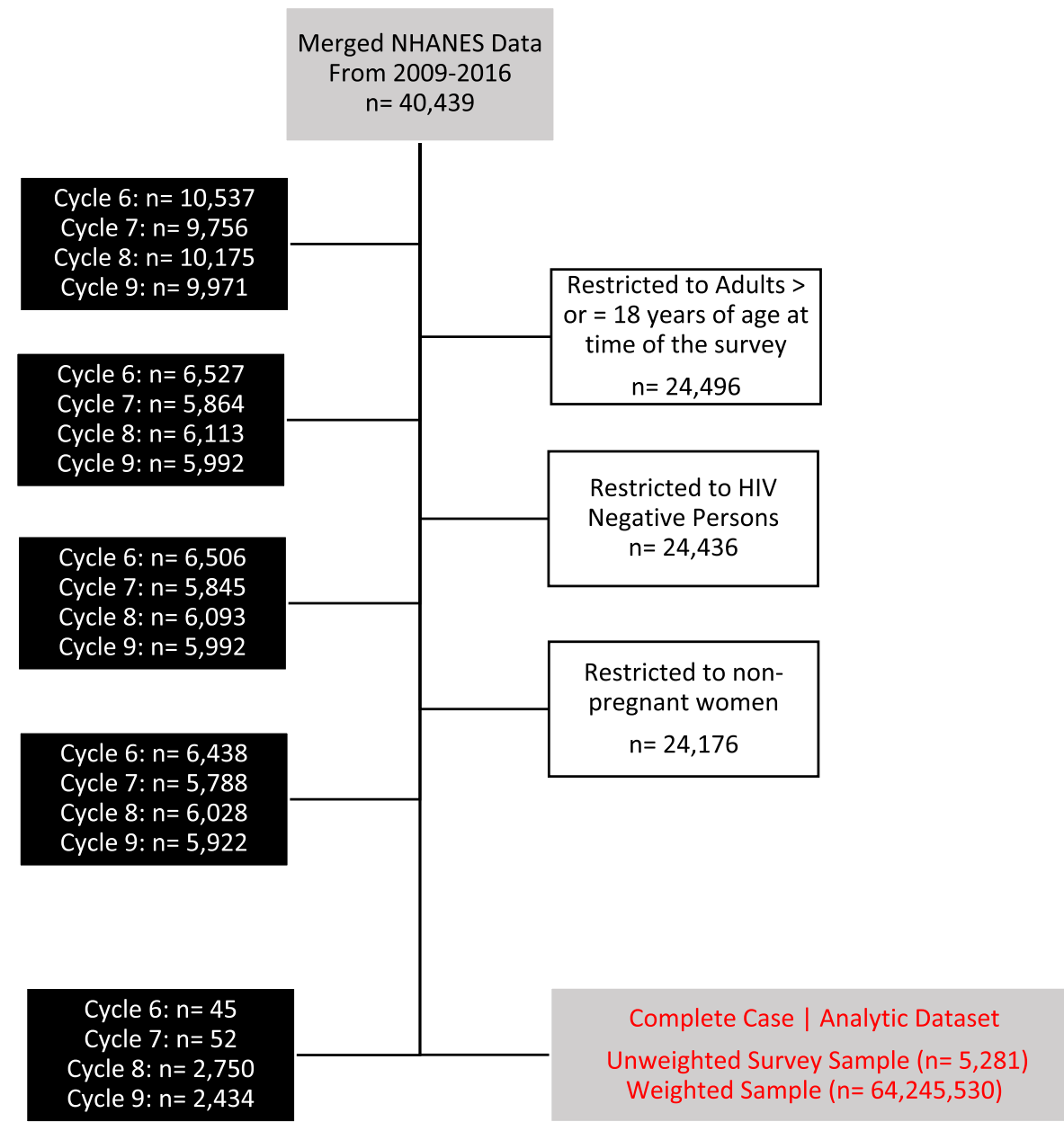

Fig. 1 Illustration of the exclusion criteria applied to generate an analytic dataset from a merger of national health and nutrition examination survey cycle years 2009 to 2016. The described exclusion criteria were applied for the following reasons: HIV infection depletes the innate immune system and may affect monocyte count; pregnancy was considered to affect female complete blood cell counts and the dataset was restricted to subjects $\geq 18$ years of age as complete blood cell counts differ by developmental stage. Cycle refers to the two-year NHANES data cycle (SDDSRVYR) from which the data was retrieved. Following application of the exclusion criteria, $n=18,895$ observations were omitted as they lacked complete case information across 15 variables. The complete case analytic dataset contains $n=5281$ unweighted observations from the survey sample, one-hundred and twenty-two $(n=122)$ of which were exposed to chronic HCV

model was fit to each imputed dataset to estimate the effect of chronic HCV infection on MPR. The resulting odds ratios were pooled by Rubin's rules [33]. A secondary analysis was performed in the imputed dataset, using the R packages: mice and mitools.

\section{Sensitivity analysis}

The sensitivity of the primary and secondary analysis was evaluated by weighted propensity score analysis [34]. The propensity scores were generated by tenfold cross-validated ensemble machine learning. The super learner was specified to predict by: a general linear model, elastic net, bootstrap aggregation and classification, regression training [35]. The ensembled propensity scores were applied by inverse proportional weighting to estimate the average treatment effect [36]. Weights were scaled by the mean and truncated by the first and ninety-ninetieth percentile. Changes in covariate balance after propensity score weighting was measured by reduction of standardized mean difference (SMD) [36]. Covariates unbalanced by propensity score weighting (SMD > $0.2)$ were adjusted for in the outcome model. Sensitivity analysis was performed in the complete case dataset, using the $\mathrm{R}$ packages: superlearner and cobalt. All statistical analysis was conducted in R version 3.6.1 [37].

\section{Results}

\section{Statistical analysis}

Transformation of the monocyte-to-platelet ratio (MPR)

The MPR was calculated from the NHANES variables "LBDMONO" and "LBXPLTSI" and converted to 1000 cells $/ \mathrm{ml}$. In the analytic dataset, the value of MPR ranges from $0.27-22.22$ with a mean of 2.53 
Table 1 Structure and characteristics of the complete case dataset from the National Health and Nutrition Examination Survey study period, 2009-2006 to investigate the effect of chronic hepatitis C virus infection on the monocyte-to-platelet ratio

\begin{tabular}{|c|c|c|c|c|c|}
\hline Variable Name & Level & Total & Low MPR & High MPR & Rao-Scott $X^{\wedge} 2^{a}$ \\
\hline & . & $5281[64,245,530]^{\mathrm{b}}$ & $2754(0.54)$ & $2527(0.45)$ & \\
\hline Chronic HCV = Positive (\%) & & 122 & $42(0.90)$ & $80(2.5)$ & $<0.001$ \\
\hline Age (mean $[S D]$ ) & . & . & $42.74(13.64)$ & $43.93(14.36)$ & 0.054 \\
\hline Sex $=$ Male & . & 2759 & $1220(40.3)$ & $1539(65.1)$ & $<0.001$ \\
\hline \multirow[t]{5}{*}{ Race (\%) } & . & & & & $<0.001$ \\
\hline & Black & 1079 & $626(10.9)$ & $453(9.0)$ & \\
\hline & White & 2127 & $1091(65.4)$ & $1036(71.3)$ & \\
\hline & Hispanic & 1377 & $797(15.4)$ & $580(13.5)$ & \\
\hline & Other & 698 & $455(8.30)$ & $243(6.20)$ & \\
\hline \multirow[t]{5}{*}{ General Health (\%) } & & & & & 0.022 \\
\hline & Excellent & 462 & $277(10.80)$ & $185(8.80)$ & \\
\hline & Very Good & 1487 & $877(35.30)$ & $610(32.30)$ & \\
\hline & Good & 3188 & $1747(52.40)$ & $1441(56.60)$ & \\
\hline & Poor & 144 & $68(1.50)$ & $76(2.30)$ & \\
\hline Transfusion = Yes (\%) & . & 427 & $242(7.80)$ & $185(8.3)$ & 0.723 \\
\hline Needle Use $=$ Yes (\%) & & 146 & $63(2.60)$ & $83(3.10)$ & 0.479 \\
\hline Married = Lives Alone (\%) & . & 2094 & $1202(36.0)$ & $892(34.50)$ & 0.312 \\
\hline \multirow[t]{4}{*}{ Social Economic Status (\%) } & . & & & & 0.88 \\
\hline & $\leq 1.30$ & 1650 & $887(20.70)$ & $763(21.80)$ & \\
\hline & $\leq 1.85$ & 699 & $396(11.10)$ & $303(10.80)$ & \\
\hline & $>1.85$ & 2824 & $1624(66.3)$ & $1200(65.5)$ & \\
\hline White Blood Cell Count (mean [SD]) & & . & $6.81(1.93)$ & $8.00(2.34)$ & $<0.001$ \\
\hline Cancer Diagnosis = Yes (\%) & . & 311 & $186(8.70)$ & $125(6.90)$ & 0.028 \\
\hline Anemia $=$ Yes (\%) & & 515 & $117(3.70)$ & $62(2.10)$ & 0.006 \\
\hline \multirow[t]{4}{*}{ Diabetes Diagnosis (\%) } & & & & & 0.003 \\
\hline & Not Diabetic & 4663 & $2672(92.2)$ & $1991(88.6)$ & \\
\hline & Pre-Diabetic & 103 & $49(1.40)$ & $54(2.30)$ & \\
\hline & Diabetic & 515 & $248(6.40)$ & $267(9.10)$ & \\
\hline Average Alcohol Consumption per Day (mean [SD]) & . & . & $3.30(29.13)$ & $3.54(15.22)$ & 0.789 \\
\hline
\end{tabular}

${ }^{a}$ The Rao-Scott Chi Square test was used to test for independence between each of the described covariates and monocyte-to-platelet ratio in survey weighted data $(a=0.05)$

Percentages represent the unweighted population of the NHANES study base

$\mathrm{b}$ the weighted survey sample number of participants with complete case data in the study

and standard deviation of 1.05. Linear regression found a positive association between chronic $\mathrm{HCV}$ infection and MPR. MPR was categorized with a cut point of 2.49, Low (min-2.49), High (> 2.49-max). Logistic regression was used to evaluate the accuracy of this cut point in combination with a receiver operator characteristic curve analysis (sensitivity of $65 \%$ and a specificity of $54 \%$ ) (Additional file 1: Figure S3).

\section{Descriptive statistics}

The analytic dataset includes $n=5281(n=64,245,530$ after weighting the sample) observations with $n=122(n=1,067$, 882 sample weighted) exposures to chronic HCV infection, the outcome was recorded for all exposures (Table 1). Bivariate analysis of the relationship between chronic $\mathrm{HCV}$ infection and MPR found a positive correlation, $66 \%$ of persons with chronic HCV infection have a high MPR $(>2.49)(P<$ 0.001) (Table 1). Characteristics of the analytic sample were determined by unweighted survey-sample number and as a weighted sample percentage, representing the population percentage (Table 1).

\section{Statistical inference}

Primary analysis

A survey-weighted logistic regression model was built by use of a DAG and AIC backward elimination to 
estimate the effect of chronic HCV infection on MPR with adjustment of potential confounders (Table 2). Notably, none of the covariates which did not meet the definition of a confounder were found to increase model fit or precision of the effect estimates during automated variable selection. All the confounders illustrated in the DAG were included throughout automated variable selection. The variables chronic $\mathrm{HCV}$ infection, age, sex, race, white blood cell count (WBC) and cancer diagnosis exhibit a statistically significant relationship with MPR $(P<0.05)$. No variables were removed from the parsimonious model selected by Akaike information criterion. No collinearity was detected with race having the greatest VIF value equal to 3.77 . The model selected for primary analysis (Table 2) estimates that those chronically infected with HCV have 3.10 times the odds of a high MPR score than those not exposed $(\mathrm{OR}=3.10$, [95\%CI: $1.55-6.18]$ ).

\section{Secondary analysis}

The association between exposure to chronic $\mathrm{HCV}$ infection and MPR was also investigated by multiple imputation of the covariate needle use. The minimum correlation matrix selected: age, transfusion status, sex, monocyte count, platelet count, cancer, and diabetes as predictors of needle use. A total of seven hundred and sixty-eight observations $(n=768)$ were added by multiple imputation, including thirteen additional exposures $(n=13)$. The outcome (MPR) was recorded for all the imputed data. Combined estimates from the ten imputed datasets show that those chronically infected with $\mathrm{HCV}$ have 2.79 times the odds of a high MPR score than those not exposed (OR $=2.79$, [95\%CI: 1.43-5.46]) (Fig. 2).

\section{Sensitivity analysis}

The sensitivity of the primary and secondary analysis was tested by propensity score weighting. Propensity scores were predicted for $\mathrm{HCV}$ exposure by ensemble machine learning. The standardized mean differences of the covariates: age, race, sex, general health status, transfusion status, needle use, marital status, social-economic status did not meet the $<0.2$ cutoff; therefore, they were adjusted for in the outcome model. Propensity score weighting estimated that those chronically infected with $\mathrm{HCV}$ have 2.24 times the odds of a high MPR score than those not exposed (OR $=2.24$, [95\%CI: 1.31-3.84]) (Fig. 2).

We do not interpret effect estimates for variables other than the primary exposure as doing so, may require

Table 2 Results of survey weighted logistic regression analysis in the relationship between chronic hepatitis $C$ infection and categorized monocyte-to-platelet ratio (low, high): from a complete case dataset of the National Health and Nutrition Examination Survey, 2009-2016

\begin{tabular}{|c|c|c|c|c|}
\hline Variables & Reference & Level & Adjusted Odds Ratio & Confidence Interval $^{\mathrm{a}}$ \\
\hline \multirow[t]{2}{*}{ Chronic HCV } & Negative & . & & \\
\hline & . & Positive & 3.10 & $1.55-6.18$ \\
\hline Age & . & . & 1.01 & $1.00-1.02$ \\
\hline \multirow[t]{4}{*}{ Race } & Black & . & & \\
\hline & . & White & 1.12 & $0.93-1.36$ \\
\hline & & Hispanic & 0.78 & $0.62-0.98$ \\
\hline & . & Other & 0.78 & $0.60-1.01$ \\
\hline \multirow[t]{2}{*}{ Sex } & Female & & & \\
\hline & . & Male & 3.35 & $2.89-3.88$ \\
\hline \multirow[t]{2}{*}{ Needle Use } & No & . & & \\
\hline & . & Yes & 0.63 & $0.35-1.13$ \\
\hline \multirow[t]{3}{*}{ Diabetes } & Not Diabetic & . & & \\
\hline & . & Pre-Diabetic & 1.77 & $0.90-3.47$ \\
\hline & . & Diabetic & 1.06 & $0.79-1.43$ \\
\hline \multirow[t]{2}{*}{ Cancer } & No Diagnosis & & & \\
\hline & . & Diagnosis & 0.74 & $0.56-0.99$ \\
\hline WBC & & . & 1.39 & $1.33-1.45$ \\
\hline
\end{tabular}

a the $95 \%$ confidence interval for the given estimate

Multi-collinearity was tested by the variable inflation factor, no inflation was detected $<5$ 


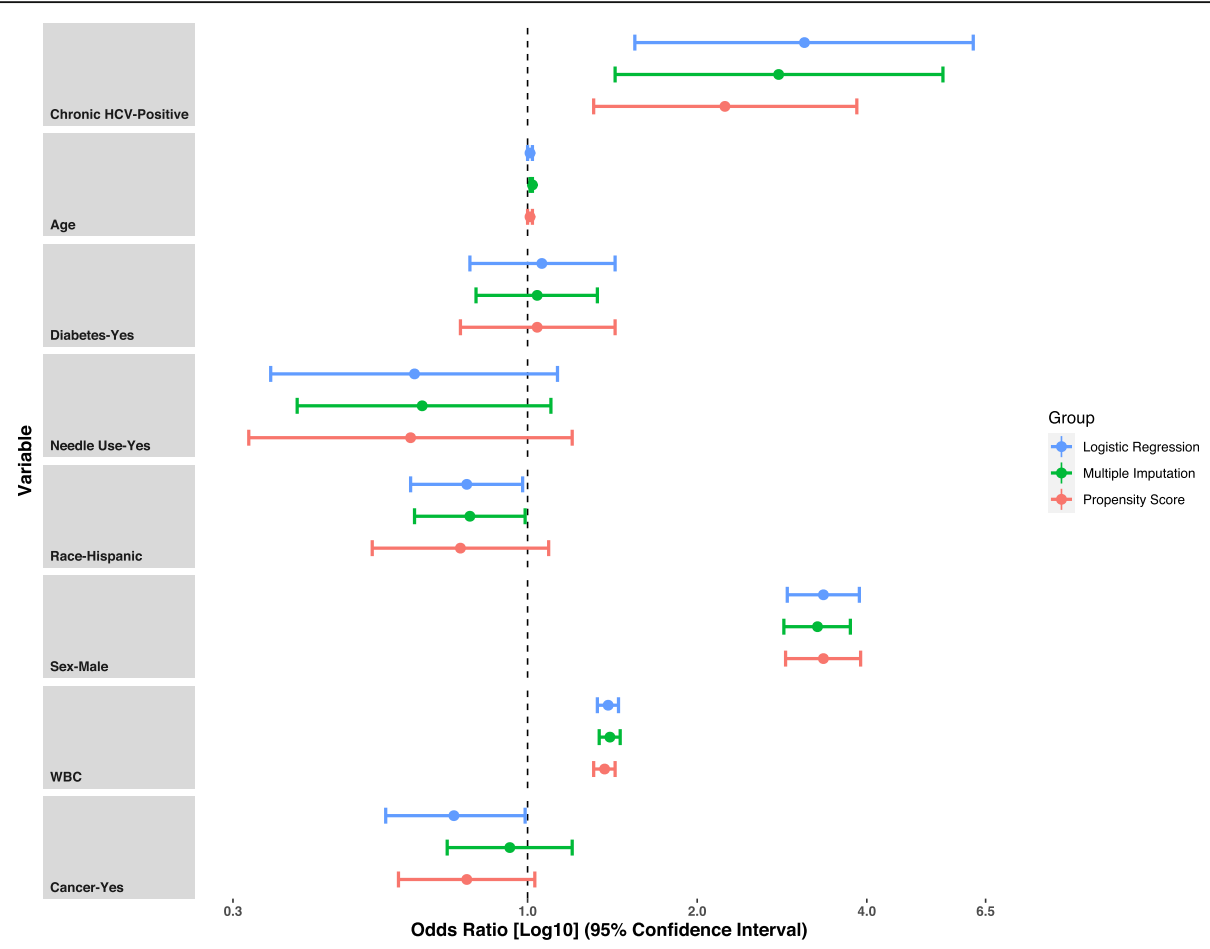

Fig. 2 Results of survey weighted analysis examining the relationship between chronic Hepatitis C infection and monocyte-to-platelet ratio in complete case and missing data from the National Health and Nutrition Examination Survey cycle years 2009 to 2016. The adjusted odds ratio and $95 \%$ confidence intervals shown in the log scale for statistically significant covariates in the primary analysis, secondary analysis or those requiring adjustment after propensity score weighting as a sensitivity analysis. All analyses incorporated survey features from the medical examination center derived weight following the methodology of Ridgeway et al. [34]

specification of a separate causal framework to account for additional confounding [38].

\section{Discussion}

\section{Summary of results}

The primary analysis found a strong measure of association between chronic HCV infection and MPR in the population of the United States, after adjusting for the confounders: age, race, sex, needle use, diabetes, white blood cell count and cancer. Americans with chronic HCV infection have 3.10 times or $210 \%$ higher odds of a high MPR score, than those not exposed. The adjusted effects of age, sex and needle use agree with known literature, exposure to injectable drugs was found to decrease monocyte count in a mouse model and within a cohort of opioid users $[39,40]$. The secondary and sensitivity analysis agreed with the primary results, suggesting that MPR differs in those chronically infected and not infected with $\mathrm{HCV}$.

\section{Comparison with literature}

Past studies describe an association between chronic HCV infection and low platelet count [11, 41]. The mechanism of this relationship remains poorly understood. The virus may trigger auto-immunogenicity and decrease the production of thrombopoietin [42].

Chronic HCV infection affects monocyte count through stimulation of the innate and adaptive immune response [13]. Monocytes differentiate into dendritic cells or macrophage in the human immune system. $\mathrm{HCV}$ infects both cell types, which serve as reservoirs for viral replication in vivo [43]. Conceptually, monocyte count may indicate the presence and severity of $\mathrm{HCV}$ infection.

Tsai et al. [14] analyzed the effect of chronic HCV infection on blood cell counts adjusting for the demographic confounders of age and sex. They found that monocyte count positively, and platelet count negatively associated with $\mathrm{HCV}$ viral load. Monocyte count increased by one-thousand cells per microliter in patients who seroconverted.

Streiff et al. [12] conducted an NHANES study to understand peripheral blood count abnormalities in patients exposed to HCV. An association was found between $\mathrm{HCV}$ exposure and platelet counts at cutoff $(\mathrm{OR}=2.33$, [95\% CI: $1.08-5.02]$; no relationship was found for monocyte count.

In summary, monocyte count increases and platelet count decreases in response to chronic HCV infection, 
as the odds of a high MPR were greater in those exposed. The population-level differences in MPR between those infected and not infected with HCV in the United States make it a candidate screening tool for chronic HCV infection. Performance of the MPR as a screening tool depends on its predictive accuracy (sensitivity/ specificity), the prevalence of $\mathrm{HCV}$ in the target population and availability of other tests in the resource setting. Theoretically, implementation of the MPR as a screening tool for chronic HCV infection may have the most impact in a low-resource, high-prevalence setting where clinicians could leverage previously reported completeblood-count data before follow-up with a secondary screening test or a confirmatory NAAT type diagnostic.

\section{Strengths and limitations}

The presented study possesses strength in design, analysis, and interpretability. The single temporal collection point of cross-sectional studies evokes the issue of reverse causality [44]. In the NHANES sample, variable characteristics were collected by means of an interview or through the medical examination center (MEC) [15]. The natural history of HCV infection establishes temporal causality in the relationship with complete blood cell counts. The MEC tested for HCV-RNA by NAAT and the incubation period of HCV lasts between 2 to 26 weeks with an average of 7 weeks [45]. Therefore, if a person tests $\mathrm{HCV}$ positive at the MEC they were initially exposed to the virus at least 2 weeks before, establishing unidirectional causality.

Two additional analyses were conducted (multiple imputation and propensity score weighting) both support the robustness of a positive relationship between chronic $\mathrm{HCV}$ infection and MPR. The variable needle use was imputed because, it meets the criterion of missing at random [33]. Persons who inject drugs are less likely to report doing so to a federal agency than to report no needle use [46]. Therefore, imputation served to help estimate a more accurate rate of needle use in the population of the United States. In addition to reducing bias, missing data analysis also aids precision and increases statistical power [33]. Weighted propensity score analysis allows estimation of the average treatment effect (ATE), a measure commonly deployed in randomized clinical trials where the control and treatment groups exhibit exchangeability. In cross-sectional studies, ATE allows estimation of treatment effect when the observed groups have a similar probability of exposure to the selected confounders [47]. In studies on biomarkers, confounding by indication due to the effect of a drug prescribed for a comorbidity can alter the distribution of a marker in those treated [48]. Propensity score weighting partially accommodates for this bias, as the comorbidity serves in the surrogate of confounding from the drug. The exposed and non-exposed groups have a more equal probability of exposure to the drug through the similar probability of exposure to the indication [49]. The use of NHANES data across several collection years benefits the ability to generalize from and the validity of our study. Past analysis of NHANES data has found true population-level effects such as the role of high cholesterol in heart disease [15].

Weaknesses of the study include the potential for unmeasured confounding, such as co-infection. Risk factors for HCV exposure overlap with those of other chronic infectious diseases like hepatitis B and tuberculosis [50]. Laboratory data confirming active infection with these pathogens was not publicly available in the NHANES dataset [15]. The variable white blood cell count (WBC) was selected as a measure of proxy confounding, with the conceptual understanding that exposure to a foreign antigen increases WBC [51]. The possibility also exists that co-infection acts as an independent predictor or mediates the effect of chronic HCV infection on MPR [52]. Due to the cross-sectional study design, it was not possible to evaluate how the duration of $\mathrm{HCV}$ infection affects MPR. In the age of interferon-based therapy, Ikeda et al. [53] found that low platelet count, before the initiation of treatment, was a significant risk factor in the development of hepatocellular carcinoma (HCC) during follow-up. The ability of low platelet count to predict late-stage liver pathology in those with HCV infection means that thrombocytopenia may occur earlier in the disease process than liver fibrosis or HCC [53].

\section{Clinical and epidemiological interpretation}

Analysis by logistic regression adjusted for the confounding variables: age, race, biological sex, needle use, white blood cell count, cancer diagnosis, and diabetes all share a dependent relationship with MPR. The relationship between these confounders and platelet count is unexplainable because, expected platelet counts are not well described for healthy populations [14, 54]. The cut point to categorize MPR in our study can be interpreted in the range of normal complete blood counts in the United States. The division of the lower normal limit of expected monocyte count by platelet count produces a MPR of approximately 1.33 [55]. We caution overinterpretation of this value as true population-level counts of monocytes and platelets are not well understood and can vary. An MPR of 1.33 could be derived from counts below or above the normal range.

\section{Future directions}

Future analysis should focus on investigating the relationship between chronic HCV infection and MPR in a country other than the United States. Geographical validation would strengthen the conclusion that chronic 
HCV infection affects MPR. Unmeasured confounding should also be assessed by adjustment for relevant coinfections and prescription of immune-modulating drugs. A logical follow up to this work would be the use of the MPR alone or in combination with other risk factors to build a predictive model to screen for chronic $\mathrm{HCV}$ infection. We caution that a biomarker like the MPR should not be used independently for diagnosis of $\mathrm{HCV}$ infection but in combination with a specific serological or NAAT type test. Implementation of the MPR in a reflex testing algorithm where a "high" score would requisition a follow up test could benefit the positive predictive value of diagnosis, conserve tests in resource limited settings and make population level screening initiatives economically viable. The primary benefit of population level screening with the MPR being that it would enhance surveillance for $\mathrm{HCV}$ infection. Persons that may not otherwise be tested for $\mathrm{HCV}$, could be identified from a complete blood count. Subsequent research efforts should test the diagnostic accuracy of the MPR in a study population, or work to identify additional complete blood count-based measurementswhich are associated with chronic HCV infection- and may increase the discrimination of MPR as a predictive tool.

\section{Conclusion}

A relationship exists between chronic $\mathrm{HCV}$ infection and the complete blood count biomarker (monocyte-toplatelet ratio) in the population of the United States. We conclude that generally chronic HCV infection increases monocyte count and decreases platelet count. Understanding the relationship between chronic HCV and MPR, could advise a biomarker-based screening tool for chronic HCV infection, similar to the use of the APRI for liver fibrosis [9]. Development of an antigen free, biomarker-based, screening tool for chronic HCV infection would have tremendous economic benefits and decrease the time-to-treatment through the initiation of care from a complete blood draw.

\section{Supplementary Information}

The online version contains supplementary material available at https://doi. org/10.1186/s12889-021-11267-w.

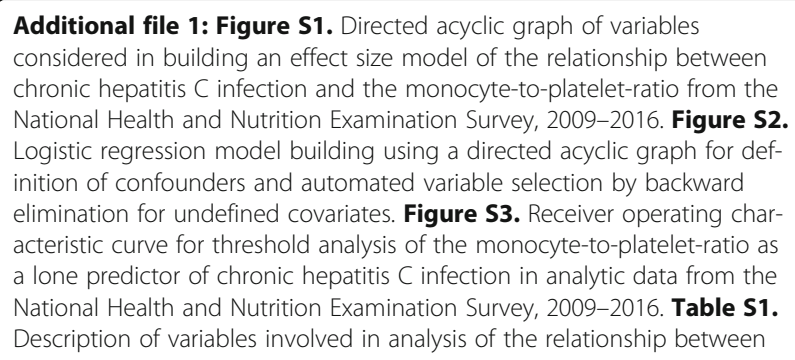

chronic hepatitis $C$ infection and the monocyte-to-platelet ratio from the National Health and Nutrition Examination Survey, 2009-2016.

\section{Acknowledgments \\ The authors thank NHANES participants and staff for their contributions to the database.}

\begin{abstract}
Authors' contributions
All authors helped conceive and design the study. AMN and MEK performed the analysis, DMP and ANJ contributed their expertise in epidemiology and clinical virology. The manuscript was written by AMN. All authors interpreted the analysis and edited the manuscript. The author(s) read and approved the final manuscript.
\end{abstract}

\section{Funding}

A.M.N (under the supervision of D.M.P and A.N.J) was awarded a Frederick Banting and Charles Best Canada Graduate Scholarship from the Canadian Institutes of Health Research to pursue doctoral studies (\#434951). A.M.N also received funding from the University of British Columbia Public Scholars Initiative for completion of this work.

M.E.K. is supported in part by a Scholar Award from the Michael Smith Foundation for Health Research, partnered with Centre for Health Evaluation and Outcome Sciences (ID\#: 17661), and receives research funding from the Discovery Grant and Discovery Launch Supplement by the Natural Sciences and Engineering Research Council (PG\#: 20 R01603 and 20R12709; PI: M.E.K.). Over the past 3 years, M.E.K. has received consulting fees from Biogen (unrelated to the current project) and participated in Advisory Boards and/or Satellite Symposia of Biogen Inc.

\section{Availability of data and materials}

The datasets analyzed in the described study are available as part of the NHANES repository, https://wwwn.cdc.gov/nchs/nhanes/Default.aspx.

\section{Declarations}

\section{Ethics approval and consent to participate}

The University of British Columbia's Policy 89, item 7.10.3 on studies involving human participants and Article 2.2 of the Tri-Council Policy Statement on Ethical Conduct for Research Involving Humans, provide ethical support of the study. As described in both policies research ethics board approval is not required for studies involving publicly available data such as that of NHANES. NHANES is a publicly available dataset administered by the National Center for Health Statistics, written informed consent was provided by all participants to the study investigators. The authors adhered to ethical principles, described in the declaration of Helsinki, and followed ethical guidelines or regulations provided by the University of British Columbia.

Consent for publication

Not applicable, as all presented data is de-identified.

\section{Competing interests}

The authors have no competing interests.

\section{Author details}

${ }^{1}$ School of Population and Public Health, University of British Columbia, Vancouver, British Columbia V6T 1Z4, Canada. ${ }^{2}$ British Columbia Centre for Disease Control Public Health Laboratory, Virology, Provincial Health Services Authority, Vancouver, British Columbia V5Z 4R4, Canada. ${ }^{3}$ Centre for Health Evaluation and Outcome Sciences, Providence Health Care, Vancouver, British Columbia V6Z 1Y6, Canada. 'British Columbia Centre for Disease Control, Communicable Diseases and Immunization Services, Provincial Health Services Authority, Vancouver, British Columbia V5Z 4R4, Canada. ${ }^{5}$ Department of Pathology and Laboratory Medicine, University of British Columbia, Vancouver, British Columbia V6T 1Z4, Canada. 
Received: 25 November 2020 Accepted: 9 June 2021 Published online: 13 July 2021

\section{References}

1. World Health Organisation. Global hepatitis report, 2017. Geneva; 2017. https://www.who.int/hepatitis/publications/global-hepatitis-report2017/en/.

2. World Health Organization. Hepatitis C. Fact Sheet. 2019. https://www.who. int/news-room/fact-sheets/detail/hepatitis-c. Accessed 8 Jul 2019.

3. Canadian Network on Hepatitis C. Blueprint to Inform Hepatitis C Elimination Efforts in Canada. 2019. https:/pacificaidsnetwork.org/files/2019/ 06/blueprint_hcv_2019_05.pdf.

4. Denniston MM, Jiles RB, Drobeniuc J, Klevens RM, Ward JW, McQuillan GM, et al. Chronic hepatitis C virus infection in the United States, National Health and nutrition examination survey 2003 to 2010. Ann Intern Med. 2014;160(5):293-300. https://doi.org/10.7326/M13-1133.

5. Seo S, Silverberg MJ, Hurley LB, Ready J, Saxena V, Witt D, et al. Prevalence of spontaneous clearance of hepatitis C virus infection doubled from 1998 to 2017. Clin Gastroenterol Hepatol. 2019;18(2):511-3. https://doi.org/10.101 6/j.cgh.2019.04.035.

6. Das D, Pandya M. Recent advancement of direct-acting antiviral agents (DAAs) in hepatitis C therapy. Mini-Reviews Med Chem. 2018;18(7):584-96. https://doi.org/10.2174/1389557517666170913111930.

7. Chevaliez S. Strategies for the improvement of HCV testing and diagnosis. Expert Rev Anti-Infect Ther. 2019;17(5):341-7. https://doi.org/10.1080/14 787210.2019.1604221.

8. Aronson JK, Ferner RE. Biomarkers-A General Review. In: Current protocols in pharmacology. Hoboken: John Wiley \& Sons, Inc.; 2017. p. 9.23.1-9.23.17. doi:https://doi.org/10.1002/cpph.19.

9. Loaeza-del-Castillo A, Paz-Pineda F, Oviedo-Cárdenas E, Sánchez-Ávila F, Vargas-Vorácková F. AST to platelet ratio index (APRI) for the noninvasive evaluation of liver fibrosis. Ann Hepatol. 2008;7(4):350-7. https://doi.org/10.1 016/S1665-2681(19)31836-8.

10. National Center for Health Statistics. National Health and Nutrition Examination Survey. 2019. https://www.cdc.gov/nchs/nhanes/index.htm.

11. Kauf TL, Nelson DR, Schelfhout J, Delaney JA, Wang PF. Trends in the prevalence of thrombocytopenia among individuals ilnfected with hepatitis C virus in the United States, 1999-2008. BMC Res Notes. 2012;5(1):142. https://doi.org/10.1186/1756-0500-5-142.

12. Streiff MB, Mehta S, Thomas DL. Peripheral blood count abnormalities among patients with hepatitis $C$ in the United States. Hepatology. 2002; 35(4):947-52. https://doi.org/10.1053/jhep.2002.32486.

13. Yang Y, Tu Z-K, Liu X-K, Zhang P. Mononuclear phagocyte system in hepatitis C virus infection. World J Gastroenterol. 2018;24(44):4962-73. https://doi.org/10.3748/wjg.v24.i44.4962.

14. Tsai M-H, Lin K-H, Lin K-T, Hung C-M, Cheng H-S, Tyan Y-C, et al. Predictors for early identification of hepatitis C virus infection. Biomed Res Int. 2015; 2015:429290-7. https://doi.org/10.1155/2015/429290

15. National Center for Health Statistics. National Health and Nutrition Examination Survey: CDC/National Center for Health Statistics; 2019. https:// www.cdc.gov/nchs/nhanes/index.htm. Accessed 1 Jan 2019.

16. Wolf $\mathrm{C}$, Best $\mathrm{H}$. The SAGE handbook of regression analysis and causal inference. Los Angeles: SAGE Publications Ltd; 2014. https://uk.sagepub. com/en-gb/eur/the-sage-handbook-of-regression-analysis-and-causalinference/book238839.

17. Heeringa SG, West BT, Berglund PA. Regression with Complex Samples. In: The SAGE Handbook of Regression Analysis and Causal Inference. London: SAGE Publications Ltd. p. 225-48. https://doi.org/10.4135/9781446288146. n11.

18. Government of Canada Panel on Research Ethics. Tri-Council Policy Statement: Ethical Conduct for Research Involving Humans - TCPS 2. Policy. 2018. http://www.pre.ethics.gc.ca/eng/tcps2-eptc2_2018_chapter2-cha pitre2.html.

19. The University of British Columbia Board of Governors. Research Involving Human Participants. Policy. 2019. https://universitycounsel-2015.sites.olt.ubc. ca/files/2019/08/Human-Research-Policy_LR9.pdf.

20. Mirel L, Mohadjer L, Dohrmann S. National Health and nutrition examination survey: estimation procedures, 2007-2010. 2013. https://www.cdc.gov/nchs/ data/series/sr_02/sr02_159.pdf.

21. Roudot-Thoraval F, Bastie A, Pawlotsky J, Dhumeaux D. Epidemiological factors affecting the severity of hepatitis $C$ virus-related liver disease: a
French survey of 6,664 patients. Hepatology. 1997;26(2):485-90. https://doi. org/10.1002/hep.510260233.

22. Shepard CW, Finelli L, Alter MJ. Global epidemiology of hepatitis C virus infection. Lancet Infect Dis. 2005;5(9):558-67. https://doi.org/10.1016/S14 73-3099(05)70216-4.

23. Graf ABA, Bousquet O, Rätsch G, Schölkopf B. Prototype classification: insights from machine learning. Neural Comput. 2009;21(1):272-300. https:// doi.org/10.1162/neco.2009.01-07-443.

24. Rosch EH. Natural categories. Cogn Psychol. 1973;4(3):328-50. https://doi. org/10.1016/0010-0285(73)90017-0.

25. Rao JNK, Scott AJ. The analysis of categorical data from complex sample surveys: chi-squared tests for goodness of fit and Independence in two-way tables. J Am Stat Assoc. 1981;76(374):221-30. https://doi.org/10.1080/016214 59.1981.10477633.

26. Chou R, Cottrell EB. Screening for hepatitis $C$ virus infection in adults : a systematic review for the USPSTF. Ann Intern Med. 2013;158(2):101-8. https://www.ncbi.nlm.nih.gov/books/NBK115423/. https://doi.org/10.7326/ 0003-4819-158-2-201301150-00574.

27. Krajden M, Cook D, Janjua NZ. Contextualizing Canada's hepatitis C virus epidemic. Can Liver J. 2018;1(4):218-30. https://doi.org/10.3138/canlivj.20180011.

28. Menart-Houtermans B, Rütter R, Nowotny B, Rosenbauer J, Koliaki C, Kahl S, et al. Leukocyte profiles differ between type 1 and type 2 diabetes and are associated with metabolic phenotypes: results from the German diabetes study (GDS). Diabetes Care. 2014;37(8):2326-33. https://doi.org/10.2337/ dc14-0316.

29. VanderWeele TJ. Principles of confounder selection. Eur J Epidemiol. 2019; 34(3):211-9. https://doi.org/10.1007/s10654-019-00494-6.

30. Pearl J. Causal Diagrams for Empirical Research. Biometrika. 1995;82(4):669. https://doi.org/10.2307/2337329.

31. Lumley T, Scott A. AIC and BIC for modeling with complex survey data. J Surv Stat Methodol. 2015;3(1):1-18. https://doi.org/10.1093/jssam/smu021.

32. James G, Witten D, Hastie T, Tibshirani R. An introduction to statistical learning. New York: Springer New York; 2013. https://doi.org/10.1007/ 978-1-4614-7138-7.

33. Little RJA, Rubin DB. Statistical analysis with missing data. Hoboken: John Wiley \& Sons, Inc.; 2002. https://doi.org/10.1002/9781119013563.

34. Ridgeway G, Kovalchik SA, Griffin BA, Kabeto MU. Propensity score analysis with survey weighted data. J causal inference. 2015;3(2):237-49. https://doi. org/10.1515/jci-2014-0039.

35. Rose S. Mortality risk score prediction in an elderly population using machine learning. Am J Epidemiol. 2013;177(5):443-52. https://doi.org/10.1 093/aje/kws241.

36. Austin PC. A tutorial and case study in propensity score analysis: an application to estimating the effect of in-hospital smoking cessation counseling on mortality. Multivariate Behav Res. 2011;46(1):119-51. https:// doi.org/10.1080/00273171.2011.540480

37. Rstudio Team. RStudio. 2019. https://www.rstudio.com/products/team/.

38. Westreich D, Greenland S. The table 2 fallacy: presenting and interpreting confounder and modifier coefficients. Am J Epidemiol. 2013;177(4):292-8. https://doi.org/10.1093/aje/kws412.

39. Orum MH, Kara MZ, Egilmez OB, Kalenderoglu A. Complete blood count alterations due to the opioid use: what about the lymphocyte-related ratios, especially in monocyte to lymphocyte ratio and platelet to lymphocyte ratio? J Immunoass Immunochem. 2018;39(4):365-76. https://doi.org/10.1 080/15321819.2018.1460272.

40. Di Francesco P, Falchetti R, Gaziano R, Lanzilli G, Belogi L, Ravagnan G, et al. Differential effects of short-term or prolonged cocaine exposure on peripheral blood cells in mice. Life Sci. 1994;54(25):2015-20. https://doi. org/10.1016/0024-3205(94)90136-8

41. Wang C-S, Yao W-J, Wang S-T, Chang T-T, Chou P. Strong Association of Hepatitis C Virus (HCV) infection and thrombocytopenia: implications from a survey of a community with Hyperendemic HCV infection. Clin Infect Dis. 2004:39(6):790-6. https://doi.org/10.1086/423384

42. Dahal S, Upadhyay S, Banjade R, Dhakal P, Khanal N, Bhatt VR. Thrombocytopenia in patients with chronic hepatitis C virus infection. Mediterr J Hematol Infect Dis. 2017;9(1):e2017019. https://doi.org/10.4084/ MJHID 2017.019

43. Revie D, Salahuddin SZ. Role of macrophages and monocytes in hepatitis C virus infections. World J Gastroenterol. 2014;20(11):2777-84. https://doi.org/1 0.3748/wjg.v20.i11.2777. 
44. Rothman KJ, Greenland S. Causation and causal inference in epidemiology. Am J Public Health. 2005;95(S1):S144-50. https://doi.org/10.2105/AJPH.2004. 059204.

45. Hajarizadeh B, Grebely J, Dore GJ. Epidemiology and natural history of HCV infection. Nat Rev Gastroenterol Hepatol. 2013;10(9):553-62. https://doi. org/10.1038/nrgastro.2013.107.

46. Deryabina AP, Patnaik P, El-Sadr WM. Underreported injection drug use and its potential contribution to reported increase in sexual transmission of HIV in Kazakhstan and Kyrgyzstan. Harm Reduct J. 2019;16(1):1. https://doi.org/1 0.1186/s12954-018-0274-2.

47. Abdia Y, Kulasekera KB, Datta S, Boakye M, Kong M. Propensity scores based methods for estimating average treatment effect and average treatment effect among treated: a comparative study. Biom J. 2017;59(5):967-85. https://doi.org/10.1002/bimj.201600094.

48. Spieker AJ, Delaney JAC, McClelland RL. Evaluating the treatment effects model for estimation of cross-sectional associations between risk factors and cardiovascular biomarkers influenced by medication use. Pharmacoepidemiol Drug Saf. 2015;24(12):1286-96. https://doi.org/10.1002/pds.3876.

49. Freemantle N, Marston L, Walters K, Wood J, Reynolds MR, Petersen I. Making inferences on treatment effects from real world data: propensity scores, confounding by indication, and other perils for the unwary in observational research. BMJ. 2013;347:f6409. https://doi.org/10.1136/bmj.f6409.

50. McKee G, Butt ZA, Wong S, Salway T, Gilbert M, Wong J, et al. Syndemic characterization of HCV, HBV, and HIV co-infections in a large population based cohort study. EClinicalMedicine. 2018;4-5:99-108. https://doi.org/10.1 016/.eclinm.2018.10.006.

51. Clem A. Fundamentals of vaccine immunology. J Glob Infect Dis. 2011;3(1): 73-8. https://doi.org/10.4103/0974-777X.77299.

52. Zhang J, Feng G, Zhao Y, Zhang J, Feng L, Yang J. Association between lymphocyte-to-monocyte ratio (LMR) and the mortality of HBV-related liver cirrhosis: a retrospective cohort study. BMJ Open. 2015;5(8):e008033. https:// doi.org/10.1136/bmjopen-2015-008033.

53. Ikeda M, Fujiyama S, Tanaka M, Sata M, Ide T, Yatsuhashi H, et al. Risk factors for development of hepatocellular carcinoma in patients with chronic hepatitis C after sustained response to interferon. J Gastroenterol. 2005; 40(2):148-56. https://doi.org/10.1007/s00535-004-1519-2.

54. Biino G, Santimone I, Minelli C, Sorice R, Frongia B, Traglia M, et al. Age- and sex-related variations in platelet count in Italy: a proposal of reference ranges based on 40987 subjects' data. PLoS One. 2013;8(1):e54289. https:/ doi.org/10.1371/journal.pone.0054289.

55. National Health Service. Haematology Normal Adult Reference Ranges. RWT Pathology Services. 2019. https://www.royalwolverhampton.nhs.uk/services/ service-directory-a-z/pathology-services/departments/haematology/haema tology-normal-adult-reference-ranges/.

\section{Publisher's Note}

Springer Nature remains neutral with regard to jurisdictional claims in published maps and institutional affiliations.

Ready to submit your research? Choose BMC and benefit from:

- fast, convenient online submission

- thorough peer review by experienced researchers in your field

- rapid publication on acceptance

- support for research data, including large and complex data types

- gold Open Access which fosters wider collaboration and increased citations

- maximum visibility for your research: over $100 \mathrm{M}$ website views per year

At BMC, research is always in progress.

Learn more biomedcentral.com/submissions 